\title{
Exciton Energy Transfer in Hybrid Organics-Semiconductor Nanostructure
}

\author{
O. A. Dubovskiy, V. M. Agranovich \\ Institute of Spectroscopy, Russian Academy of Science, Moscow, Russia \\ Email: dubovskiyoa@mail.ru
}

How to cite this paper: Dubovskiy, O.A. and Agranovich, V.M. (2019) Exciton Energy Transfer in Hybrid Organics-Semiconductor Nanostructure. Soft Nanoscience Letters, 9, 17-33.

https://doi.org/10.4236/snl.2019.92002

Received: April 5, 2019

Accepted: April 27, 2019

Published: April 30, 2019

Copyright ( 2019 by author(s) and Scientific Research Publishing Inc. This work is licensed under the Creative Commons Attribution International License (CC BY 4.0).

http://creativecommons.org/licenses/by/4.0/

(c) (i) Open Access

\begin{abstract}
We consider a hybrid heterostructure containing an inorganic quantum well in close proximity with organic material as overlayer. The resonant optical pumping of Frenkel exciton can lead to an efficient indirect pumping of Wannier excitons. As organic material in such a hybrid structure, we consider crystalline tetracene. In tetracene, the singlet exciton energy is close to twice the one of triplet exciton state and singlet exciton fission into two triplets can be efficient. This process in tetracene is thermally activated and we investigate here how the temperature-dependent exciton energy transfer affects the functional properties of hybrid organic-inorganic nanostructures. We have obtained the exact analytical solution of diffusion equation for organics at different temperatures defining different diffusion lengths of excitons. The effectiveness of energy transfer in hybrid with tetracene was calculated by definite method for two selected temperatures that open possibility to operate in full region of temperatures. Temperature dependence of energy transfer opens a new possibility to turn on and off the indirect pumping due to energy transfer from the organic subsystem to the inorganic subsystem.
\end{abstract}

\section{Keywords}

Hybrid Heterostructure, Frenkel Exciton, Exciton Energy

\section{Introduction}

Optical properties of hybrid organic - nonorganic nanostructures for different applications have attracted interest in theory and experiments [1]-[7]. Numerous publications of mentioned hybrid structures including the system of Wannier and Frenkel exciton states and the transfer energy from a very thin layer of semiconductor to the layer of organics were demonstrated. However, we consider now a different situation, assuming that in hybrid structure the pumping 
takes place from layer of organics to very thin layer of semiconductor. Up to now this regime has been realized only for photonic crystal [2]. Below we will consider a thin layer of tetracene which can give a stronger pumping to quantum well $(\mathrm{QW})$ in semiconductor layer. Due to temperature dependence of diffusion length of Frenkel excitons in organics appears the possibility to control the pumping efficiency by varying the sample temperature. The resonant absorption of light by thin QW and the direct creation of excitons and e-h pairs in QW are small. However the exciton density and subsequent nonlinearity in hybrid structure such as the one sketched in Figure 1 can be increased due to strong absorption of incident light by the organic overlayer having large oscillators strength followed by efficient non radiating energy transfer to inorganic QW. Such indirect pumping of QW can be very efficient if the light from external pumping beam will resonantly generate Frenkel excitons in the organic overlayer with energies significantly larger than the exciton energy in the semiconductor QW. The light absorption in the organic component of the hybrid structure can be much larger than the direct absorption by thin semiconductor QW so the organic component plays, in this case, the role of the funnel of radiation energy pumping. Following this non radiating energy transfer and subsequent energy relaxation, the QW in the hybrid structure can reach a state with a high excitation density and thus exhibit a number of nonlinear optical effects. The QW Wannier exciton line can be observed even at room temperature. Such optical nonlinearities would be relevant for a probe beam of light resonant with the

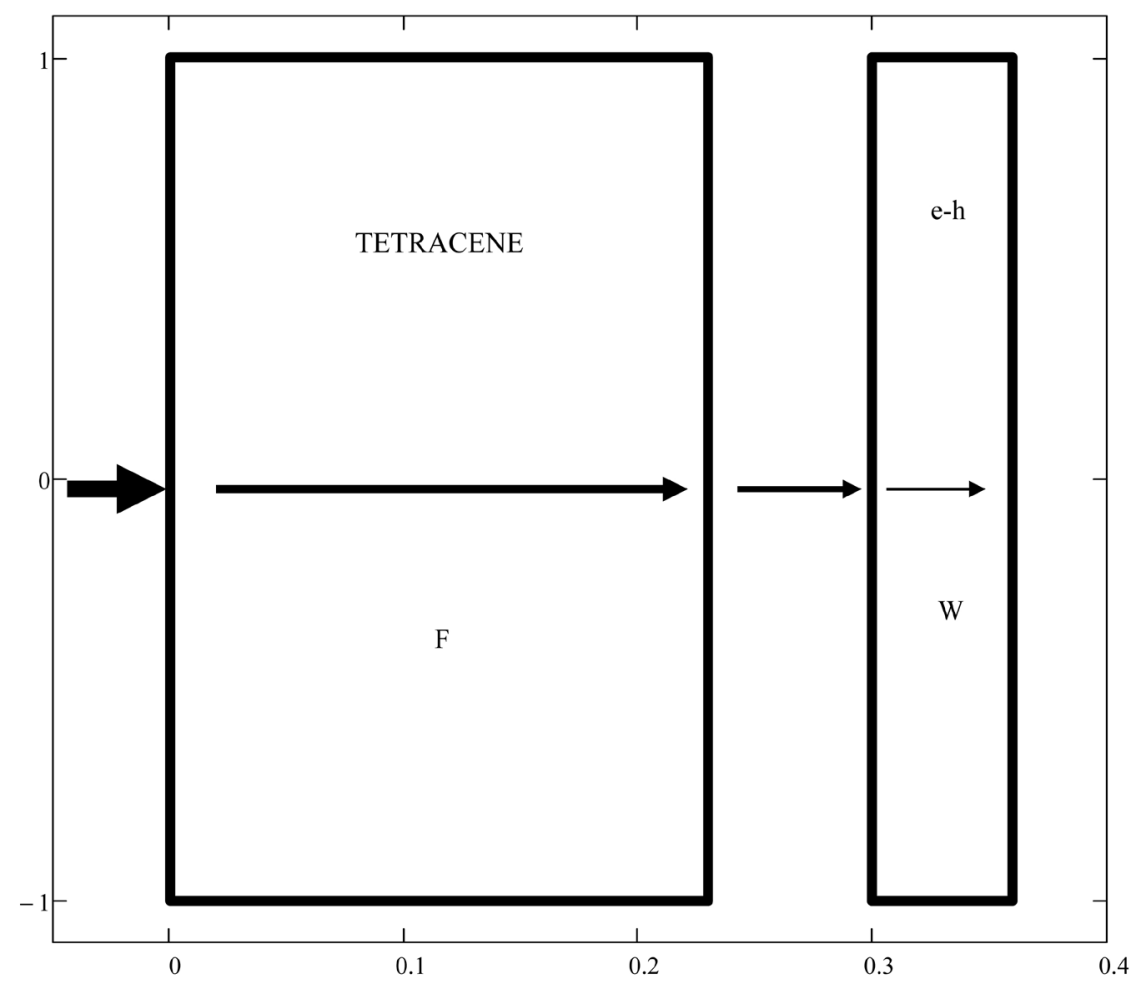

Figure 1. Scheme of hybrid with tetracene as organics with Frenkel excitons (F) and semiconductor (e-h) with Wannier excitons (W). 
QW exciton transition. As this probe has a frequency lower than the Frenkel exciton energy in the organic overlayer, it would be not concentrated in the organic part of the hybrid structure. Variations in the pumping intensity of the organic component would produce, via non radiating energy transfer to the inorganic $\mathrm{QW}$, variations in the concentration of Wannier excitons in the QW, and thus optical control of the QW nonlinearity can be achieved.

We calculate here the efficiency of such indirect optical pumping of tetracene. The parameters describing tetracene and its particular role in the thermally activated singlet exciton fission into two triplets, are taken from the experimental study by Laubel and Baessler [8] [9]. Fission of a single tetracene exciton into pair of triplet exciton reduces the singlet exciton diffusion length and for this reason, the singlet diffusion length is strongly temperature-dependent. This, in turn, opens the possibilities of an unusual temperature dependence of the energy transfer from the tetracene thin layer to semiconductor QW.

\section{Kinetics of Energy Transfer in Tetracene as Overlayer}

As organic subsystem in the hybrid structure, we consider an overlayer of crystalline tetracene. The numerical data for tetracene were taken from [8] in the temperature range $100 \mathrm{~K}<T<300 \mathrm{~K}$ with diffusion length in the interval $100 \AA<L_{D}<500 \AA$. The absorption coefficient $\alpha$ and length of the absorption $L_{P}$ are constant $L_{P}=1 / \alpha=500 \AA$. The diffusion length $L_{D}$ of singlet excitons in crystalline tetracene at room temperature is $L_{D}=120 \pm 10 \AA$. It increases exponentially with decreasing temperature until it approaches a constant value of $L_{D}=580 \pm 50 \AA$ below $100 \mathrm{~K}$. This dependence is shown in Figure 2. The temperature dependence of the singlet exciton life time $\tau$ determined by external fission into two triplet excitations can fully account for the observed temperature dependence of diffusion length. In fact, the exciton diffusion coefficient in crystalline tetracene is $D=L_{D}^{2} / \tau=3.3 \times 10^{-3} \mathrm{~cm}^{2} \cdot \mathrm{s}^{-1}$ and it is with high accuracy independent of temperature. Dependence of the diffusion length on temperature leads to the possibility of indirect pumping II-VI QW via energy transfer from the tetracene layer in the hybrid nanostructure of Figure 1. To undergo fission the exciton singlet in tetracene needs to obtain an additional energy of about $0.175 \mathrm{eV}$ from the interaction with lattice vibration and for this reason the life-time of exciton in tetracene strongly decreases with increasing temperature. This in turns leads to a strong dependence of the diffusion length on temperature. Thus, in order to obtain an efficient indirect pumping at a given temperature, it is necessary to use the tetracene layer thickness $L$ close to the diffusion length $L_{D}$ at this temperature. Otherwise, the energy transfer from tetracene to the II-VI QW will be suppressed because due to fission only triplet excitons and not singlet excitons will be able to reach the barrier of the QW. These remarks are fixing following restriction that for a given tetracene thickness $L$ the indirect pumping via singlet excitons can be efficient only in a restricted temperature interval up to critical temperature which decreases with increasing $L$. 


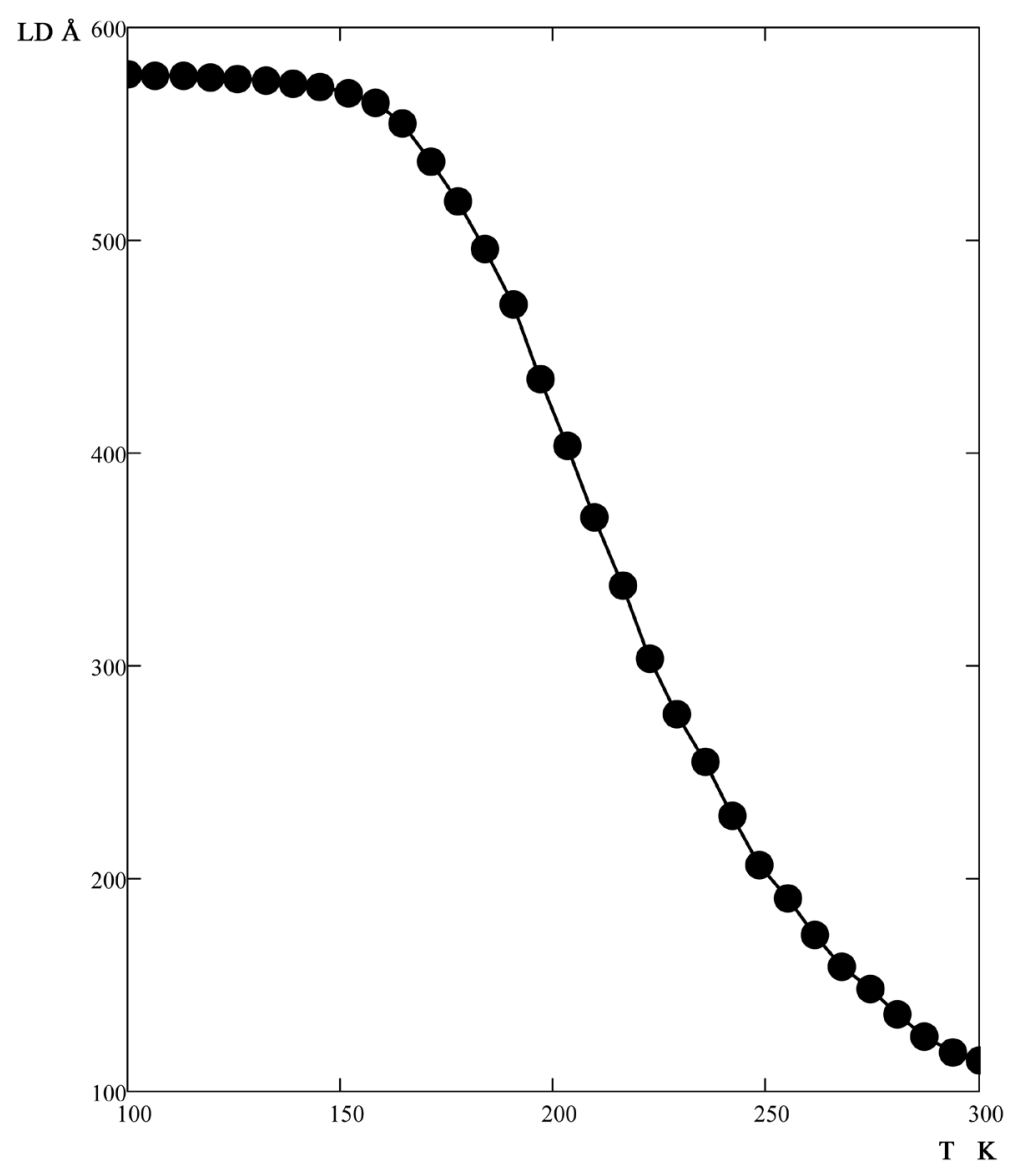

Figure 2. Dependence of exciton diffusion length from temperature [8].

The diffusion kinetic equation for organics in range $0<x<L$ has the following form in accordance with [4]

$$
L_{D}^{2} \frac{\mathrm{d}^{2} \rho}{\mathrm{d} x^{2}}=\rho-I_{0} \alpha \tau \mathrm{e}^{-\alpha x}
$$

In Equation (1), $I_{0}$ is the external radiation flux from region $x<0$. In order to solve the Equation (1) of second order, the corresponding boundary condition on the boundary $x=0$ is necessary. The boundary condition of continuity for energy flow in the organic matter and the external flow on the inlet border is the kinetic representation for the mechanism of conversion for a flow of light in region $x<0$ to flow of energy, i.e. flow of excitons in organics. This boundary equation, corresponding in dimension to the Equation (1) has the following form

$$
-\frac{1}{\alpha^{2} \tau}\left(\frac{\mathrm{d} \rho(x)}{\mathrm{d} x}\right)_{x=0}=I_{0}
$$

The solution of the linear Equation (1) should include the spatial dependence determined both by the external spatially attenuated "influence" in the right part (1), and the spatial dependence corresponding to the "own" solution at zero external influence. In this case, the proper "own" solution should be also spatially 
attenuated. These two solutions are presented in general form with factors A and B as follows

$$
\rho(x)=A \mathrm{e}^{-\alpha x}+B \mathrm{e}^{-\frac{x}{L_{D}}}
$$

Substitution (3) in (1) due to the corresponding cancellation of the "own" solution leads to the following definition for factor $A$

$$
A=I_{0} \alpha \tau \frac{1}{1-\left(\alpha L_{D}\right)^{2}}
$$

Substituting the ratio (4) for $A$ in border Equation (2), we obtain the following definition of the factor $B$

$$
B=-I_{0} \alpha \tau \frac{\left(\alpha L_{D}\right)^{3}}{1-\left(\alpha L_{D}\right)^{2}}
$$

As a result, substituting (4) and (5) in (3), we obtain solution in the region $0<x<L$ and $\rho(0)$ in the following form

$$
\begin{aligned}
& \rho(x)=I_{0} \alpha \tau \frac{1}{\left[1-\left(\alpha L_{D}\right)^{2}\right]}\left[\mathrm{e}^{-\alpha x}-\left(\alpha L_{D}\right)^{3} \mathrm{e}^{-\frac{x}{L_{D}}}\right] \\
& \rho(0)=I_{0} \alpha \tau \frac{1}{\left[1+\left(\alpha L_{D}\right)\right]}\left[1+\left(\alpha L_{D}\right)+\left(\alpha L_{D}\right)^{2}\right]
\end{aligned}
$$

Diffusion coefficient of tetracene $D=L_{D}^{2} / \tau=3.3 \times 10^{-3} \mathrm{~cm}^{2} / \mathrm{sec}$ and $\alpha=1 / L_{p}$, $L_{p}=0.5 \times 10^{-5} \mathrm{~cm}$ practically do not depend on temperature in region $\alpha L_{D}<1$ [8]. So we can distinguish the dependence on temperature in the factor $\alpha \tau$ as follows

$$
\alpha \tau=\left(\frac{1}{v}\right)\left(\frac{L_{D}}{L_{p}}\right)^{2}, v=\frac{D}{L_{p}}=6.6 \times 10^{3} \mathrm{~cm} / \mathrm{sec}
$$

Then substituting (7) in (6) gives complete solution in the region $0<x<L$ and $\rho(0)$ in the following form

$$
\begin{aligned}
& \rho(x)=I_{0} \frac{1}{v}\left(\alpha L_{D}\right)^{2} \frac{1}{\left[1-\left(\alpha L_{D}\right)^{2}\right]}\left[\mathrm{e}^{-\alpha x}-\left(\alpha L_{D}\right)^{3} \mathrm{e}^{-\frac{x}{L_{D}}}\right] \\
& \rho(0)=I_{0} \frac{1}{v}\left(\alpha L_{D}\right)^{2} \frac{1}{\left[1+\left(\alpha L_{D}\right)\right]}\left[1+\left(\alpha L_{D}\right)+\left(\alpha L_{D}\right)^{2}\right]
\end{aligned}
$$

Using the same as in (2) definition of flows $I(x)$ inside organics at $0<x<L$ and on outlet border flux $I_{L}$ coming out of the organic area to region $x>L$ we can define them in the following forms

$$
\begin{gathered}
I(x)=-\frac{1}{\alpha^{2} \tau}\left(\frac{\mathrm{d} \rho(x)}{\mathrm{d} x}\right)=-\frac{v}{\alpha}\left(L_{P} / L_{D}\right)^{2}\left(\frac{\mathrm{d} \rho}{\mathrm{d} x}\right), 0<x<L \\
I_{L}=-\frac{1}{\alpha^{2} \tau}\left(\frac{\mathrm{d} \rho(x)}{\mathrm{d} x}\right)_{x=L}=-\frac{v}{\alpha}\left(L_{P} / L_{D}\right)^{2}\left(\frac{\mathrm{d} \rho}{\mathrm{d} x}\right)_{x=L}
\end{gathered}
$$

Substituting the dependence (8), we obtain after differentiation the following 
definition of flux inside organics at $0<x<L$ and the light flux departing from the organics to region $x>L$

$$
\begin{gathered}
I(x)=I_{0} \frac{\left[\mathrm{e}^{-\alpha x}-\left(\alpha L_{D}\right)^{2} \mathrm{e}^{-\left(x / L_{D}\right)}\right]}{1-\left(\alpha L_{D}\right)^{2}} \\
I_{L}=I_{0} \frac{\left[\mathrm{e}^{-\alpha L}-\left(\alpha L_{D}\right)^{2} \mathrm{e}^{-\left(L / L_{D}\right)}\right]}{1-\left(\alpha L_{D}\right)^{2}}
\end{gathered}
$$

Figure 3(a) demonstrates two typical dependences of the non dimensional functions $D(x)=\rho(x)\left(v / I_{0}\right)$ with $x$ in $\AA$ for temperature $T=235 \mathrm{~K}$ by the

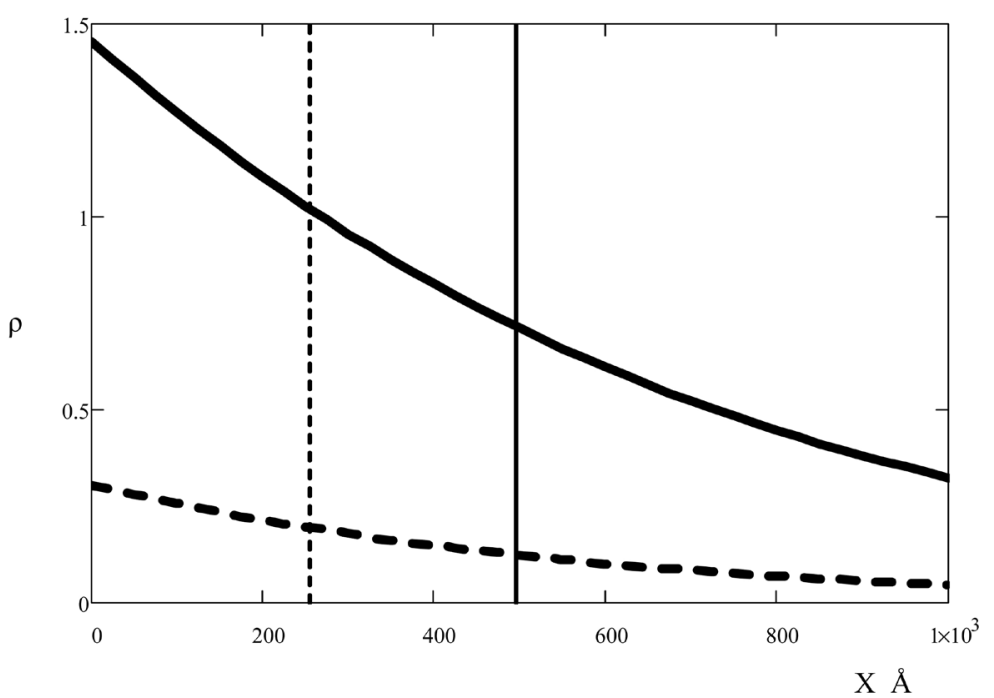

(a)

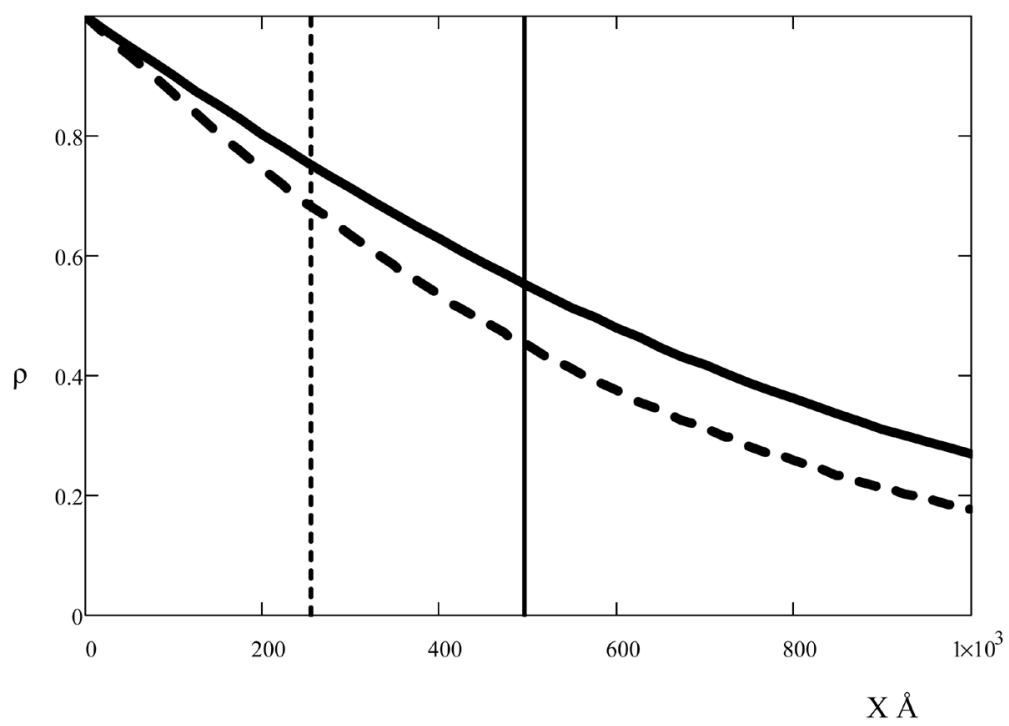

(b)

Figure 3. (a) Analytical spatial dependence $D(x)$ at $T=235 \mathrm{~K}, L_{D}=255 \AA$ (dash) and $T=184 \mathrm{~K}, L_{D}=496 \AA$ (solid); (b) Spatial dependence $F(x)$ at $T=235 \mathrm{~K}$ (dash) and $T=184 \mathrm{~K}$ (solid). 
dash curve and for lower temperature $T=184 \mathrm{~K}$ by the solid curve. The vertical dash and solid line are fixing diffusion length for these temperatures. According to Figure 2 at the first temperature, the diffusion length is equal $L_{D}=255 \AA$, and at the second lower temperature, the diffusion length is longer $L_{D}=496 \AA$. Figure 3(b) demonstrates proper to Figure 3(a) non dimensional functions $F(x)=I(x) / I_{0}$ for temperature $T=235 \mathrm{~K}$ by the dash curve and for the lower temperatures $T=184 \mathrm{~K}$ by the solid curve. It can be seen in Figure 3 that decreasing in temperature leads to a strong increase in density. Such decreasing of temperature leads also to an increase in the flow, i.e. in accordance with the definition (2) as a consequence of increasing negative derivative of density. It can be seen that the negative derivative at low temperature is much larger in relation to the negative derivative at high temperature. This increasing in density and flux is clearly determined by the inner solution of the diffusion equation with all factors-the exponent $\exp \left(-x / L_{D}\right)$, the factor $1 /\left[1-\left(\alpha L_{D}\right)^{2}\right]$ and the factor $\left(L_{D} / L_{p}\right)^{2}$.

\section{Kinetics of Energy Transfer in Hybrid with Spatially Varying Diffusion Length}

The previous part gives in kinetic approach the light flux from organic that irradiate semiconductor QW but this approach does not include distance between organic and QW, corresponding interaction between them and flux in semiconductor. It is needed to include in consideration these interactions and flux for completeness of kinetic approach. Diffusion equation includes two parameters $\tau$ and $L_{D}$ that define the transport of energy in hybrid as whole combined structure. But time of life $\tau$ in micro dynamic of excitons for crystalline systems of interacting dipoles is parameter of second order in comparison with optical molecular frequencies, energy of dipoles interaction, etc. It is defined actually by proper nonlinearities of high orders and is introduced usually as fixed phenomenological parameter. In the same time diffusion Equation (1) includes only one parameter-diffusion length $L_{D}$ that is directly defined in micro dynamics by frequencies and dipole-dipole interactions. So it is possible to investigate energy transfer in hybrid by including in consideration spatially dependent diffusion length $L_{D}^{(v)}(x)$. The region of border between organics and QW may be simulated in diffusion equation by decreasing of diffusion length in organics on this border and subsequent increasing in direction to semiconductor. It is known that diffusion length is defined by forth of dipole oscillators and distance between them. If these distances are increasing then diffusion length decreases. The border between organics and semiconductor is actually natural place for distance increasing and diffusion length decreasing. We have used for combined diffusion length $L_{D}^{(v)}(x)$ the following spatially dependence

$$
L_{D}^{(v)}(x)=L_{D}-f(x)+f(0)
$$




$$
f(x)=h\left[\frac{\left(L_{C}\right)^{2}}{\left(x-\sigma L_{D}\right)^{2}+\left(L_{C}\right)^{2}}-\frac{\left(L_{C}\right)^{2}}{\left(\sigma L_{D}\right)^{2}+\left(L_{C}\right)^{2}}\right] .
$$

This dependence distracts from diffusion length of organics $L_{D}$ additional Lorenz function with maximum at $x=\sigma L_{D}$. Then spatial dependence $L_{D}^{(v)}(x)$ has minimum at this coordinate between left organics and right semiconductor. The length $L_{C}$ defines width of Lorenz minimum and parameter h defines depth of this minimum. The additional constant term in (11) is using to conserve identity $L_{D}^{(v)}(0)=L_{D}$. These few parameters $h, \sigma, L_{C}$ were used for adjusting to hybrid structure. Small additional corrections to (11) may be used for taking into account exact tail of dependence in region of semiconductor. The solution of diffusion equation with dependence (11) will give in different regions flux between organics and semiconductor, flux in semiconductor and transmitted light flux from hybrid. Diffusion equation has now the following form

$$
\frac{\mathrm{d}^{2} \rho}{\mathrm{d} x^{2}}=\frac{1}{L_{D}^{(v)}(x)}\left(\rho-I_{0} \alpha \tau \mathrm{e}^{-\alpha x}\right)
$$

The analytical solution of diffuse equation is cumbersome and may be completely impossible, but it is no problem with computer solution for this smooth function (11) without any dangerous for computer cracks in $L_{D}^{(v)}(x)$. This function (11) with $L_{D}=400 \AA, L_{c}=100 \AA$ and $h=104, \sigma=1.2$ is demonstrated in Figure 4(a). It must be noted that dependence (11) may be used for wide regions of parameters in right side of (11) due to smooth behavior of Lorenz function in extreme. For this function with very narrow but adjusted for computer smooth peak is needed only very small computer step. Analogies of (11) may be also used for micro dynamical calculations of energy transfer shown below.

Procedure of computer solution for diffuse equation with dependence (11) by iteration method is shortly demonstrating in Appendix 1. Figure 4(b) demonstrates by dash curve the analytical dependence $D(x)=\rho(x) / \rho(0)$ at $L_{C}=0$, i.e. at constant diffusion length. Used computer algorithm was checked first for this constant diffusion length and full coincidence with analytical solution was fixed. Solid curve demonstrates the calculated dependence $D(x)$ at spatial dependence of diffuse length (11). It is seen that dash curve has monotonic decreasing. The solid curve has evident local increasing of negative derivative in region of minimum in Figure 4(a). Figure 5 demonstrates spatial dependence of correspondent fluxes $F(x)$ that specifically are derivatives of curves in Figure 4(b). The dashed and solid curves demonstrate dependences at constant and varying diffusion length. It is seen that on the left side from minimum in Figure 4(a) solid curve in Figure 5 is above dash curve. Then after smooth transfer in intermediate region it is below dash curve.

The function (11) with other parameters $L_{D}=300 \AA, L_{c}=104 \AA$ and $h=54, \sigma=1.2$ is demonstrated in Figure 6(a). The dependences $D(x)$ and $F(x)$ are shown in Figure 6(b) and Figure 7. It is seen that in comparison with Figure 4 and Figure 5 common features of spatial dependence for density and flux are the same but difference between dashed and solid curves is decreasing. 


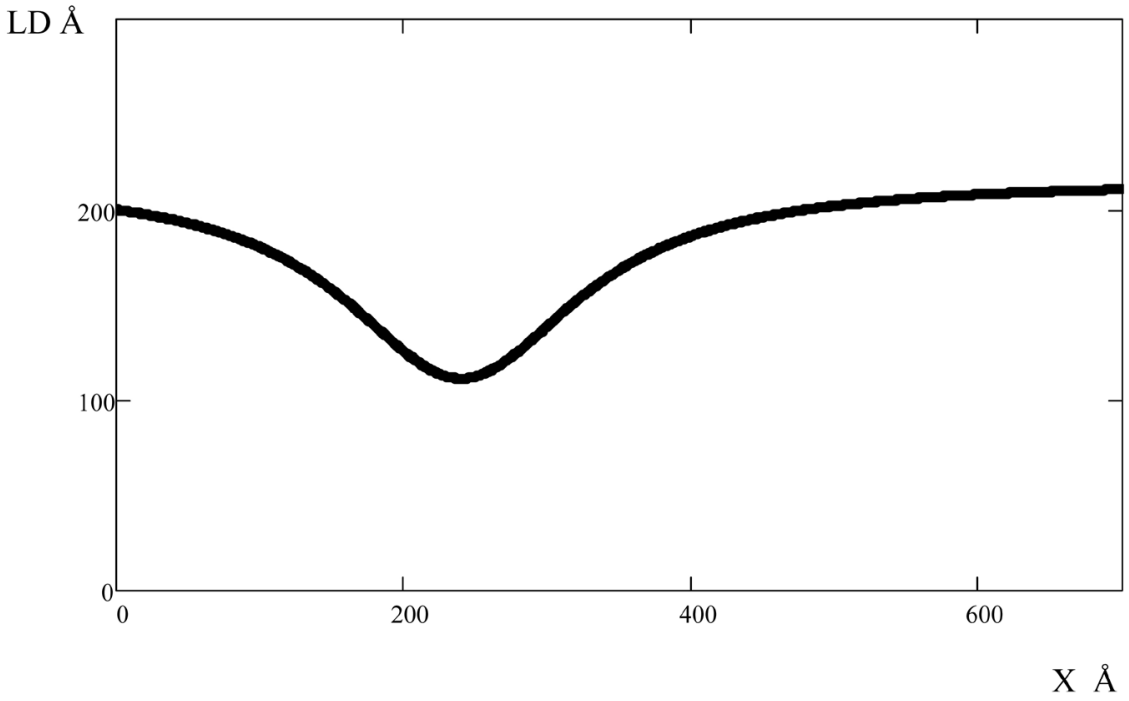

(a)

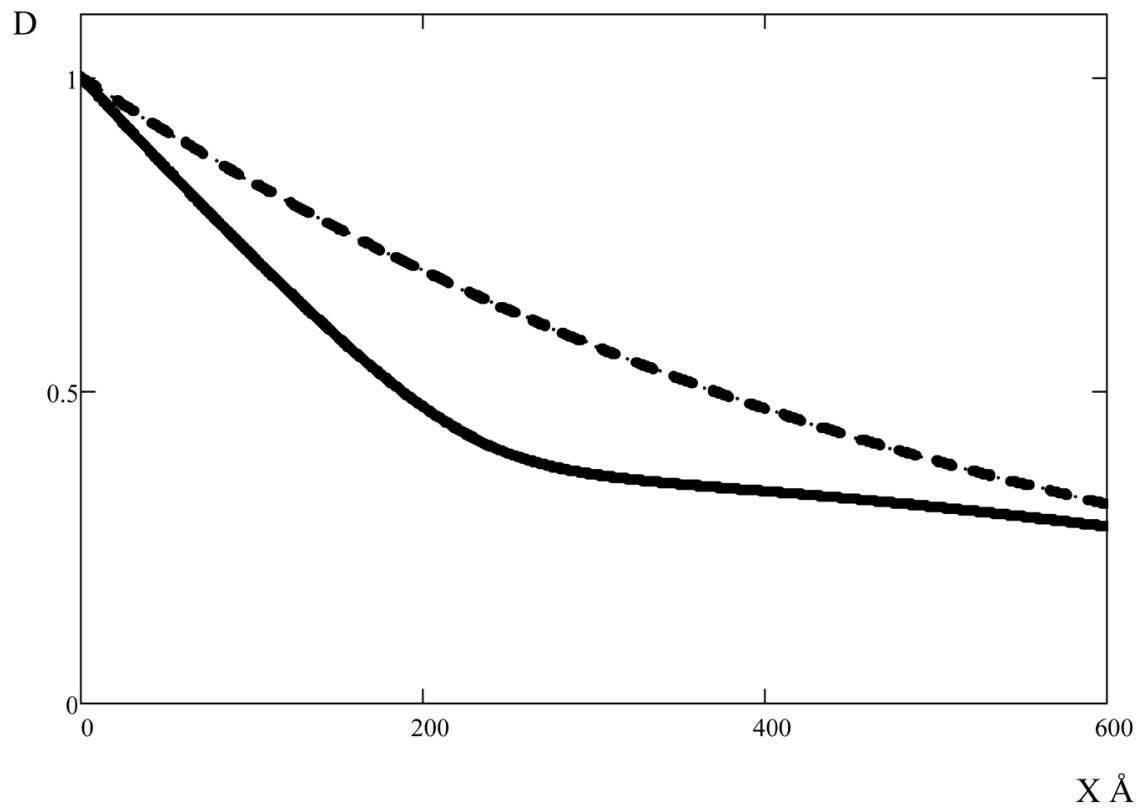

(b)

Figure 4. (a) Dependence $L_{D}^{(v)}(x)$ at $L_{D}=400 \AA, L_{c}=100 \AA, h=104, \sigma=1.2$. (b) Dashed curve demonstrates analytical dependence $D(x)$ at constant diffuse length $L_{D}=400 \AA$ and $T=200 \mathrm{~K}$. Solid curve demonstrates the calculated dependence $D(x)$ at spatial dependence of diffuse length.

The chosen set of parameters in (11) may be used for adjusting computer calculation to composed structure of real hybrids. These results demonstrate that energy transfer in whole hybrid construction may be investigated in frame of kinetic approach with diffusion equation at varying diffusion length. Estimations of energy transfer effectiveness by ratio outlet flow to inlet flow or relation exciton densities on outlet border to density on inlet border may be easily found 


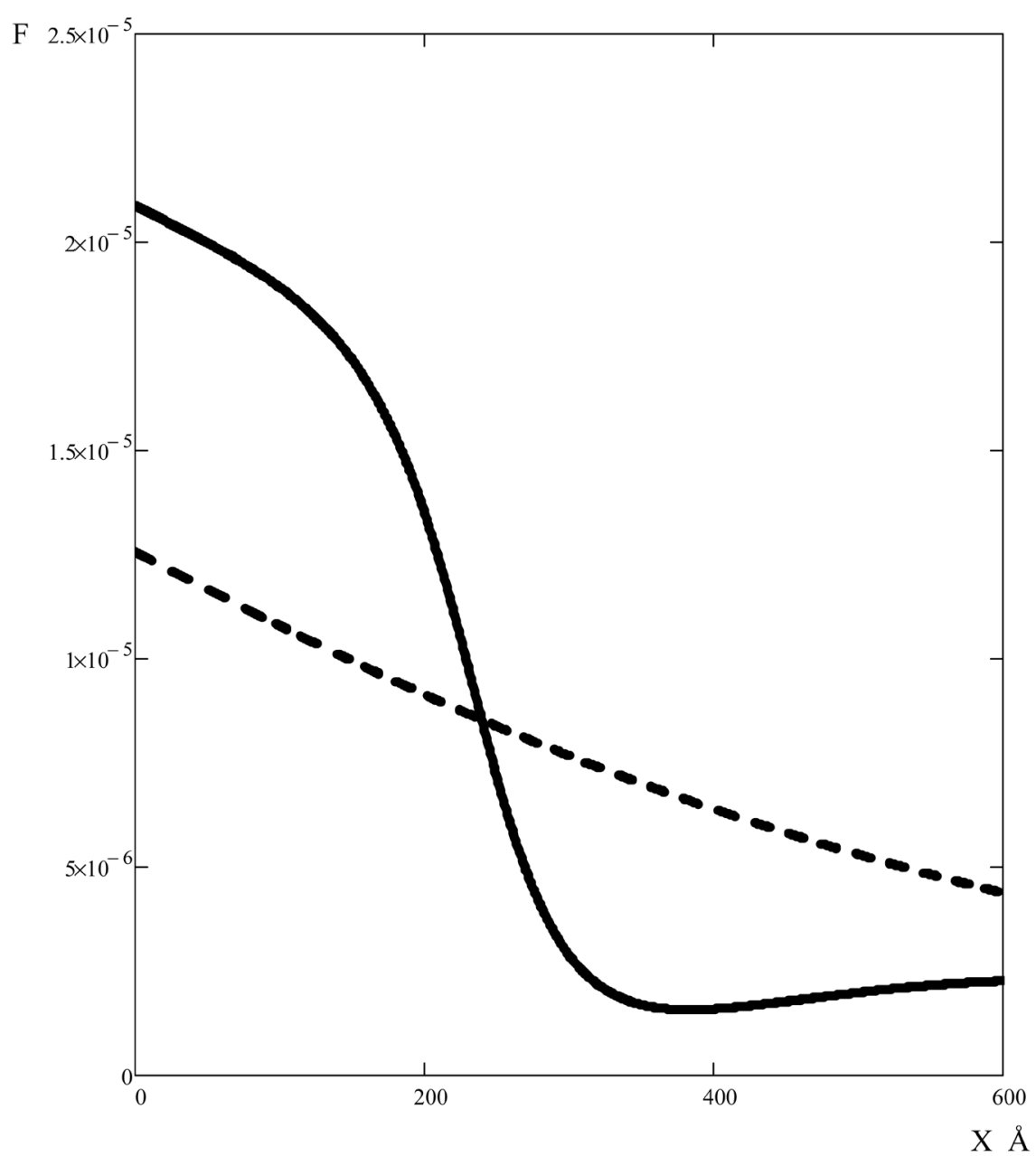

Figure 5. Spatial dependence of correspondent to Figure 4(b) fluxes $F(x)$. The dashed and solid curves demonstrate dependences at constant and varying diffusion length for the same $L_{D}=400 \AA$ and $T=200 \mathrm{~K}$.

from received results. It is evident that dependences $D(x)$ and $F(x)$ in Figure 4(b) and Figure 5 define transfer effectiveness by density and by flow Figure 5 demonstrates spatial dependence of correspondent fluxes $F(x)$ that specifically are derivatives of curves in Figure 4(b). The dashed and solid curves demonstrate dependences at constant and varying diffusion length at $L_{D}=400 \AA$ for temperature $T=200 \mathrm{~K}$. The same effectiveness is defined in Figure 6(b) and Figure 7 at $L_{D}=300 \AA$ for other temperature $T=220 \mathrm{~K}$. Analogous calculations may be performed for all regions of proper temperatures in Figure 2 corresponding to inequality $\alpha L_{D}<1$.

It will be shown in next part that micro dynamical approach gives with some important additions results that are close to that found above in kinetic approach with diffusion equation.

\section{Microdynamics of Energy Transfer in Hybrid}

Micro dynamic approach includes many dynamical parameters-frequency and 


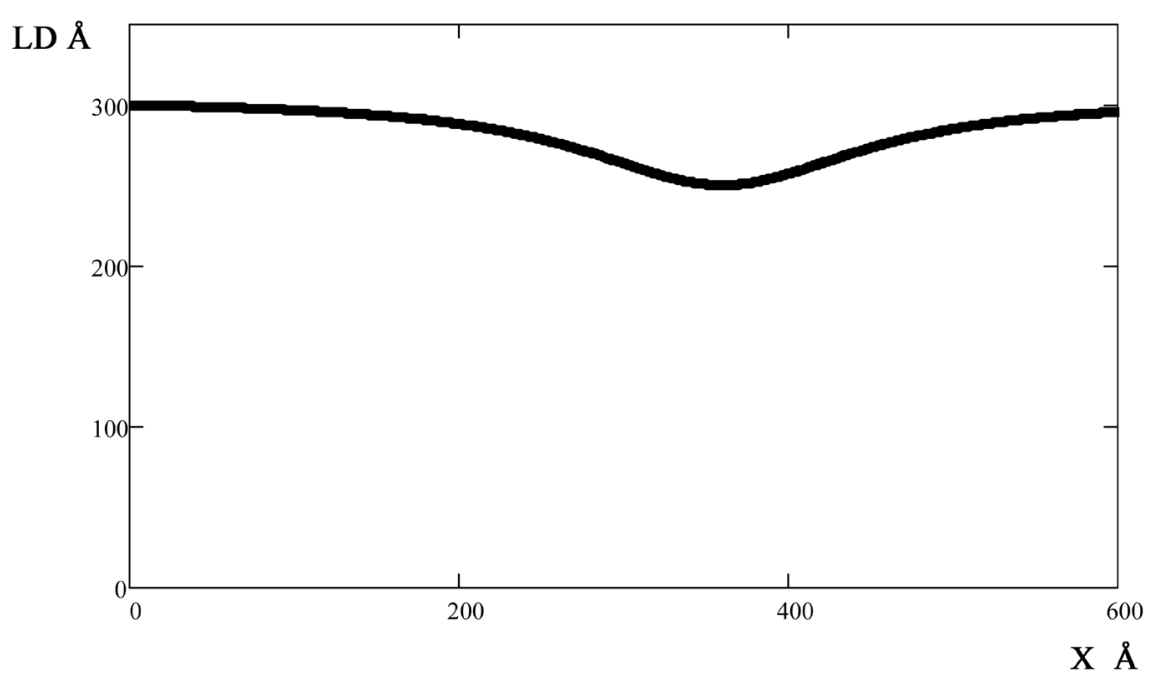

(a)

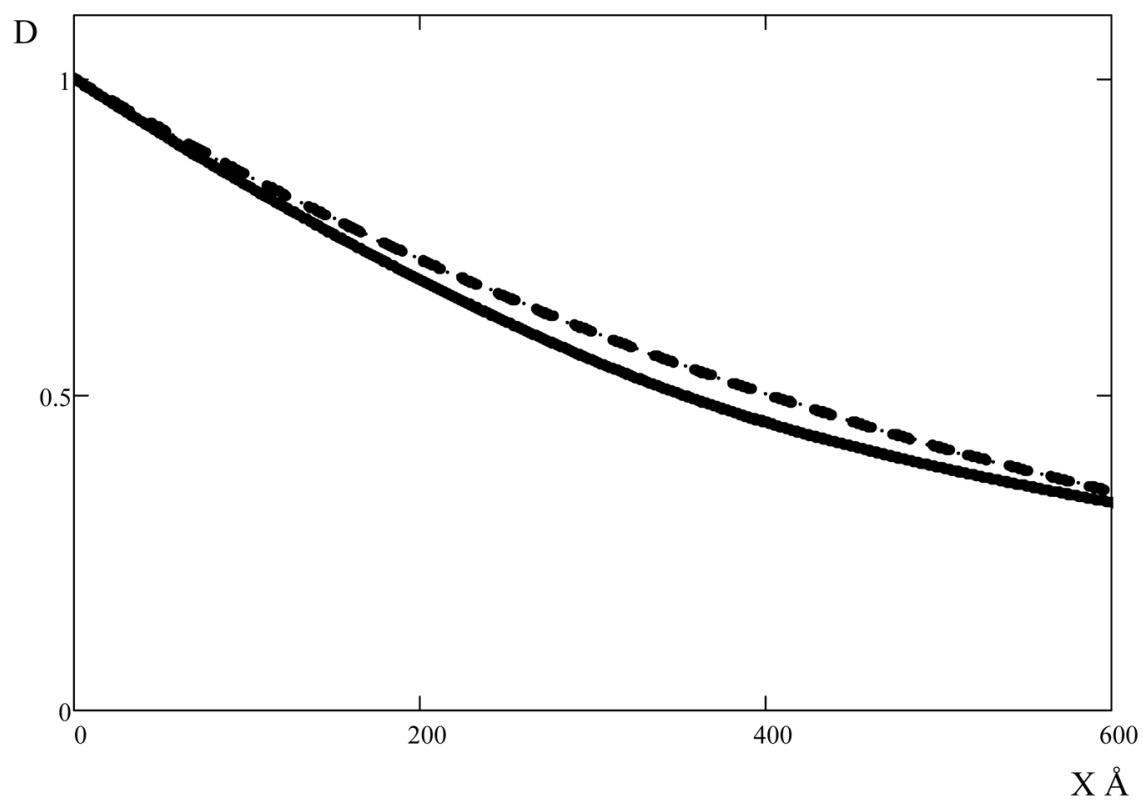

(b)

Figure 6. (a) Dependence $L_{D}^{(v)}(x)$ at parameters $L_{D}=300 \AA, L_{c}=104 \AA, h=54$, $\sigma=1.2$; (b) Dashed curve demonstrates analytical dependence $D(x)$ at constant diffuse length $L_{D}=300 \AA$ and $T=230 \mathrm{~K}$. Solid curve demonstrates the calculated dependence $D(x)$ at spatial dependence of diffusion length.

intensity of external inlet flow, own frequencies of organics and QW, Coulomb and radiation interactions inside organic, inside QW and between them. This approach is additional to kinetic approach that includes these parameters in only two kinetic parameters-external intensity and diffusion length. It is needed to demonstrate shortly more common micro dynamical process of energy transfer in hybrid organics/semiconductor that includes frequencies and interactions in composed hybrid. 


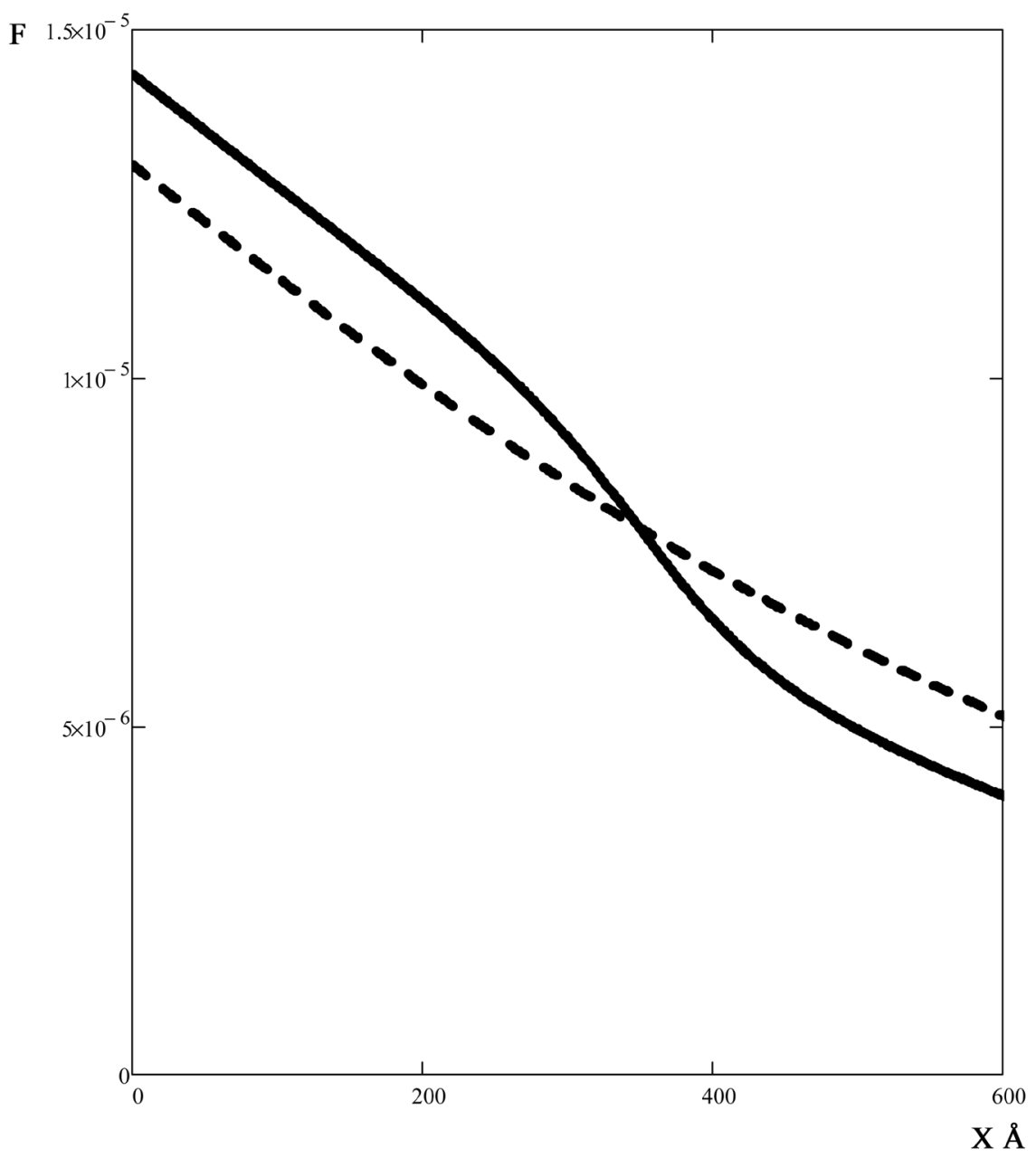

Figure 7. Spatial dependence of correspondent to Figure 6(b) fluxes $F(x)$. The dashed and solid curves demonstrate dependences at constant and varying diffusion length for the same $L_{D}=300 \AA$ and $T=230 \mathrm{~K}$.

For the simplest 1D crystal organic chain with own energy of excitons $E_{0}$ and energy of interaction between the nearest neighbors, $V$ quantum Hamilton operator of energy has the following form

$$
\hat{H}=E_{0} \sum_{n}|n\rangle\langle n|+V \sum_{n}(|n\rangle\langle n+1|+| n+1\rangle\langle n|)
$$

In relation (12) index $\mathrm{n}$ numerate organic molecules of chain. Solution of Heisenberg equation $i h(\mathrm{~d} / \mathrm{d} t) \psi=\hat{H} \psi$ may be found in the following form

$$
|\psi(t)\rangle=\sum_{n} p_{n}(t)|n\rangle
$$

The amplitudes $p_{n}$ are solution of following system of equations

$$
i h \frac{\mathrm{d}}{\mathrm{d} t} p_{n}(t)=E_{0} p_{n}(t)+V\left[p_{n+1}(t)+p_{n-1}(t)\right]
$$

We transform (14) for hybrid to following system of equations for standard dipole oscillators $p_{n}(t)=p_{n} \exp (-i \omega t)$. Then at external excitation of border 
dipole $n=0$ with intensity $I_{0}$ and frequency $\omega$ system of equations has the following form

$$
\begin{aligned}
& \omega p_{0}=\omega_{0} p_{0}+V_{0} p_{1}+I_{0} \\
& \omega p_{1}=\omega_{0} p_{1}+V_{0} p_{0}+V_{1} p_{2} \\
& \omega p_{2}=\omega_{0} p_{2}+V_{1} p_{1}+V_{2} p_{3} \\
& \vdots \\
& \omega p_{n}=\omega_{0} p_{n}+V_{n-1} p_{n-1}+V_{n} p_{n+1}, 3 \leq n \leq N-1 \\
& \vdots \\
& \omega p_{N}=\omega_{0} p_{N}+V_{N-1} p_{N-1}+V_{N}^{(W)} p_{N+1}^{(W)} \\
& \quad \omega p_{N+1}^{(W)}=\omega_{0}^{(W)} p_{N+1}^{(W)}+V_{N}^{(W)} p_{N}
\end{aligned}
$$

In (15) $\omega_{0}$ is own frequency of organics, $V_{n}$ is interaction between $n$ and $n$ +1 dipoles, frequency, dipole and interactions with QW are noted by upper index W. We shall use new original recurrent procedure of solution for equation system (15). In opposite to usual procedure, we shall suppose that amplitude of vibration of last QW amplitude $p_{N+1}^{(W)}$ is known. Then by "revert" step by step we define all other amplitudes up to the second formula in (15)

$$
\begin{aligned}
& p_{N}=\left(1 / V_{N}^{(W)}\right)\left(\omega-\omega_{0}^{(W)}\right) p_{N}^{(W)} \\
& p_{N-1}=\left(1 / V_{N-1}\right)\left[\left(\omega-\omega_{0}\right) p_{N}-V_{N}^{(W)} p_{N+1}^{(W)}\right] \\
& p_{N-2}=\left(1 / V_{N-2}\right)\left[\left(\omega-\omega_{0}\right) p_{N-1}-V_{N-1} p_{N}\right] \\
& p_{N-n}=\left(1 / V_{N-n}\right)\left[\left(\omega-\omega_{0}\right) p_{N-n+1}-V_{N-n+1} p_{N-n+2}\right], 3 \leq n \leq N-1 \\
& p_{1}=\left(1 / V_{1}\right)\left[\left(\omega-\omega_{0}\right) p_{2}-V_{2} p_{3}\right] \\
& p_{0}=\left(1 / V_{0}\right)\left[\left(\omega-\omega_{0}\right) p_{1}-V_{1} p_{2}\right]
\end{aligned}
$$

Using of the first relation in (15) gives only "definition" of $I_{0}$, that is unimportant for system of linear equations. Dividing on "found" $I_{0}$ will give only unimportant rate setting solution.

Figure 8(a) and Figure 8 (b) demonstrate the found solution of Equations (16) at following decreasing frequencies. Frequency of external excitation in non dimensional form is $\omega=1.01$. Proper frequency of organic with damping as in kinetic is $\omega_{0}=1.0-i 0.01$ and $\omega_{0}^{(w)}=0.9$ is frequency of QW. Figure 6(a) demonstrates adopted dependence for energy of interaction $V_{n}$ with maximum $V=0.15$ and minimum at $n=60$ that is analog of interaction shown in Figure 4(a) and Figure 6(a).

$$
V_{n}=V\left[1-\frac{50}{(n-60)^{2}+100}\right]
$$

Figure 8(b) demonstrates the found dependence $J_{n}=\left|p_{n}\right|^{2}, n=0 \sim 100$. It is seen that the found solution has the peculiarities of the same type that are seen in Figure 5 and Figure 7 for kinetic diffusion.

Peculiarities of dynamic in energy transfer were also investigated by classic micro dynamic of classical system that is analog to hybrid. It was considered at 


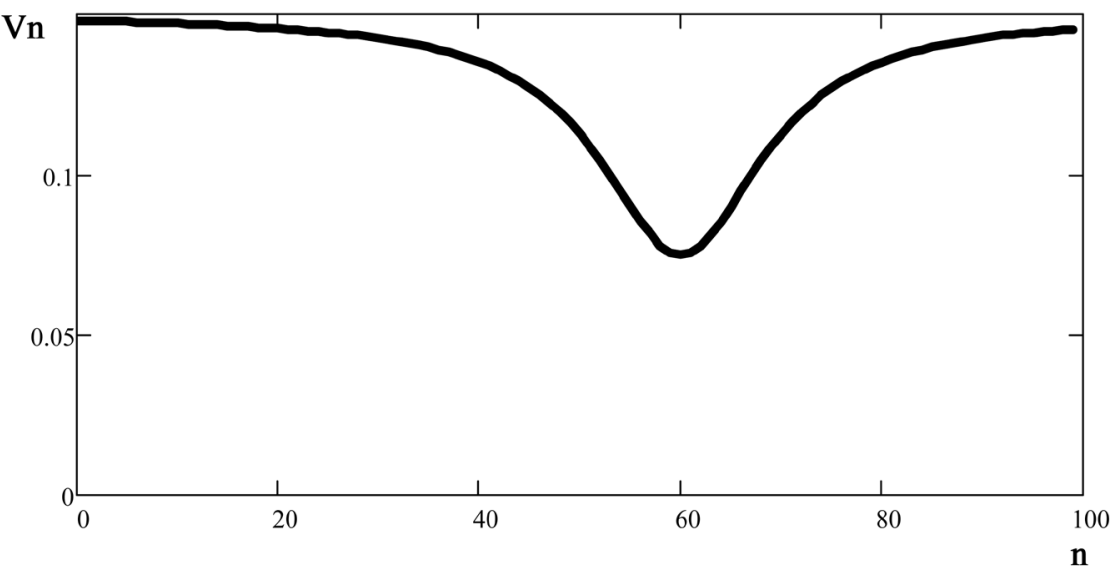

(a)

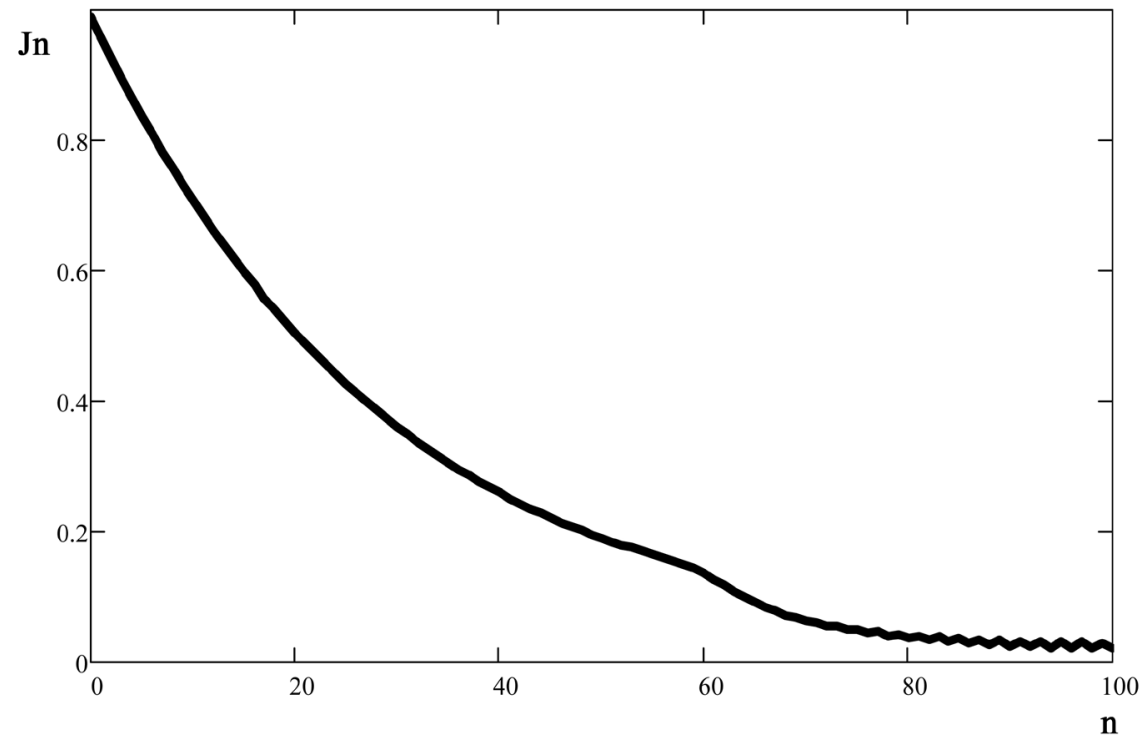

(b)

Figure 8. The found solution of Equations (16) in micro dynamic approach with $\omega=1.01, \omega_{0}=1.0-i 0.01, \omega_{0}^{(w)}=0.9$. Figure $6(\mathrm{a})$ demonstrates dependence $V_{n}$ with maximum $V=0.15$ and minimum at $n=60$. Figure $8(\mathrm{~b})$ demonstrates the found dependence $J_{n}$.

impulse excitation of 1D system with 15 identical harmonic oscillators in crystal chain with own frequency $\omega_{1}=1$ and interaction force $f_{1}=0.1$. Last oscillator of another type has different own low frequency $\omega_{2}=0.95$ and different interaction $f_{2}=0.2$ with last organic oscillator. Impulse of vibration was generated on opposite border of crystal at start. Evolution of system that was simulated on computer is solution for system of classical differential equations of second order. It is demonstrated by Figure 9 for system of 16 dipoles as set dependences from time for dipoles $P_{n}(t), n=1,2, \cdots, 15$ in non dimensional units. It is seen that wave packet of excitation moves from upper dipole marked by thick curve above to last dipole below marked also by thick curve that is analog of QW. It is seen that vibrations of intermediate dipole directly before last dipole are 


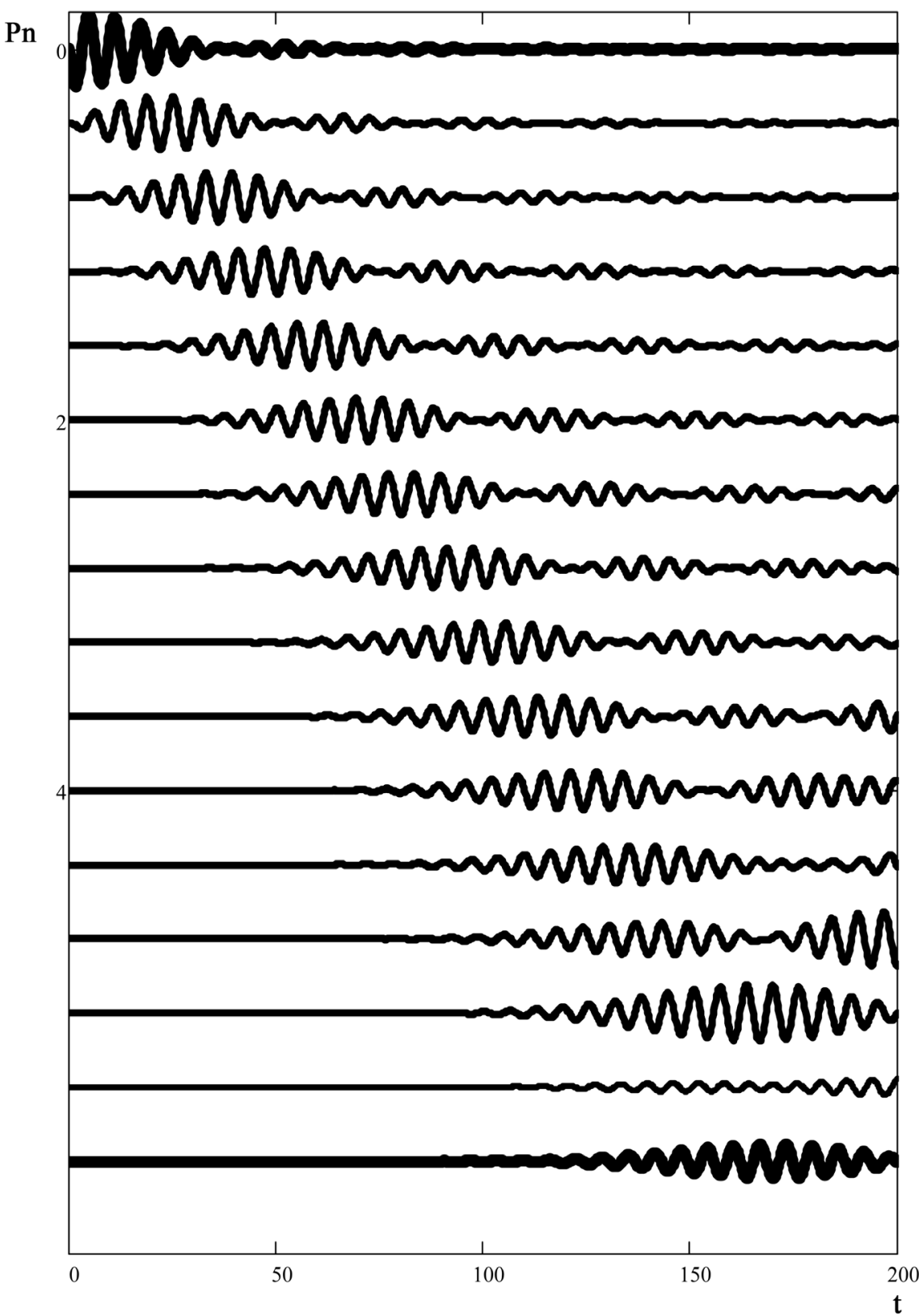

Figure 9. The set of oscillating in time dependences obtained by solution of classical equations in frame of classic micro dynamics for interacting dipoles $P_{n}(t), n=1,2, \cdots, 15$ at descending frequencies and varying interactions. Impulse of vibration was generated on upper border of crystal. . It is seen that vibrations of intermediate dipole directly before last dipole are strongly depressed in comparison with neighbor dipoles that has analogy with behavior of flow in hybrid on Figure 5 and Figure 7.

strongly depressed in comparison with neighbor dipoles. This behavior has analogy with behavior of flow in hybrid on Figure 5 and Figure 7. Between organics and QW, this effect also was seen for system of 100 dipole oscillators with continuous decreasing in proper frequencies and interaction constant that have minimum in middle region. Band of decreasing amplitudes was also seen in this middle region. 


\section{Conflicts of Interest}

The authors declare no conflicts of interest regarding the publication of this paper.

\section{References}

[1] Agranovich, V.M., Gartstein, Y.N. and Litinskaya, M. (2011) Hybrid Resonant Organic-Inorganic Nanostructures for Optoelectronic Applications. Chemical Reviews, 111, 5179-5124. https://doi.org/10.1021/cr100156x

[2] Blumstengel, S., Sadofev, S., Xu, C., Puls, J. and Henneberger, F. (2006) Converting Wannier into Frenkel Excitons in an Inorganic/Organic Hybrid Semiconductor Nanostructure. Physical Review Letters, 97, Article ID: 247401. https://doi.org/10.1103/PhysRevLett.97.237401

[3] Acherman, M., Petruska, M.A., Kos, S., et al. (2004) Energy-Transfer Pumping of Semiconductor Nanocrystals Using an Epitaxial Quantum Well. Nature, 429, 642-646. https://doi.org/10.1038/nature02571

[4] Agranovich, V.M., Basko, D.M. and La Rocca, G.C. (2012) Efficient Optical Pumping of Organic-Inorganic Heterostructures for Nonlinear Optics. Chemical Review B, 86, Article ID: 165204. https://doi.org/10.1103/PhysRevB.86.165204

[5] Blumenstegel, S., Sadofev, S., Pulset, J., et al. (2009) An Inorganic/Organic Semiconductor "Sandwich" Structure Grown by Molecular Beam Epitaxy. Advanced Materials, 21, 4850-4853. https://doi.org/10.1002/adma.200900703

[6] Itskos, G., Heliotis, G., Lagoudakis, P.G., et al. (2007) Efficient Dipole-Dipole Coupling of Mott-Wannier and Frenkel Excitons in (Ga, In)N Quantum Well/Polyfluorene Semiconductor Heterostructures. Chemical Review B, 76, 035344. https://doi.org/10.1103/PhysRevB.76.035344

[7] Zhang, Q., Atay, T., Tischler, J.R., et al. (2007) Highly Efficient Resonant Coupling of Optical Excitations in Hybrid Organic/Inorganic Semiconductor Nanostructures. Nature Nanotechnology, 2, 555-559. https://doi.org/10.1038/nnano.2007.253

[8] Laubel, G. and Baessler, H. (1970) Diffusion of Singlet Excitons in Tetracene Crystals. Molecular Crystals and Liquid Crystals, 12, 47-56. https://doi.org/10.1080/15421407008082759

[9] Agranovich, V.M., Dubovskiy, O.A., La Rocca, G.C. (2014) Singlets and Triplets in Hybrid Nanodevices. JETP Letters, 99, 323-326.

https://doi.org/10.1134/S0021364014060022 


\section{Appendix}

The relation (10) was used for definition $\rho\left(x_{0}\right)$ in initial point $x_{0}$ and $\rho\left(x_{0}+\mathrm{d} x\right)$ in next point $x_{0}+\mathrm{d} x$ at small step $\mathrm{d} x$. The iteration method use the following relation for the second derivative

$$
\frac{\mathrm{d}^{2} \rho(x)}{\mathrm{d} x^{2}}=\frac{1}{\mathrm{~d} x^{2}}[\rho(x+\mathrm{d} x)+\rho(x-\mathrm{d} x)-2 \rho(x)]
$$

This relation is reverting to the form where $\rho(x+\mathrm{d} x)$ is presenting as function of second derivative, $\rho(x)$ and $\rho(x-\mathrm{d} x)$

$$
\rho(x+\mathrm{d} x)=\mathrm{d} x^{2}\left(\frac{\mathrm{d}^{2} \rho(x)}{\mathrm{d} x^{2}}\right)_{x}+2 \rho(x)-\rho(x-\delta)
$$

So from two initial function value $\rho(\mathrm{d} x)$ and $\rho(0)$ at known second derivative we have next value $\rho(2 \mathrm{~d} x)$. Then from $\rho(\mathrm{d} x)$ and $\rho(2 \mathrm{~d} x)$ we have $\rho(3 \mathrm{~d} x)$ etc. This procedure is useful only for smooth dependences defining second derivative. 